\title{
Journal of Mathematics Research
}

\section{On the Proof of Bushell-Trustrum Inequality}

\author{
Shiqing Wang \& Yan Lou \\ College of Mathematics and Information Science \\ North China University of Water Conservancy and Electric power \\ Zhengzhou 450011, China \\ Tel: 86-371-6579-0758 E-mail: wangshiqing@ncwu.edu.cn
}

Foundation item: Support by the Natural Science Foundation of Henan (0611052600), the Basic and the Forward Position Technology Research Project of Henan.

\begin{abstract}
Bushell and Trustrum (Bushell, 1990, p. 173-178) give the famous Bushell-Trustrum inequality, but their proof exists two main mistakes which make their proof process can not establish. This paper corrects these mistakes and gives the correct proof.
\end{abstract}

Keywords: Bushell-Trustrum inequality, Positive semi-definite Hermite matrix, Unitary matrix

\section{Introduction}

Let $A$ and $B$ be two positive semi-definite Hermite matrix with rank $n$, there eigenvalues are $\lambda_{1} \geq \cdots \geq \lambda_{n} \geq 0$ and $\mu_{1} \geq \cdots \geq \mu_{n} \geq 0$, respectively. Then for any positive integer $k$, (Marcus, 1956, p. 173-174. Marshall, 1979).

$$
\sum_{i=1}^{n} \lambda_{i}^{k} \mu_{n-i+1}^{k} \leq \operatorname{tr}\left(A^{k} B^{k}\right) \leq \sum_{i=1}^{n} \lambda_{i}^{k} \mu_{i}^{k}
$$

And (Lieb and Thirring, 1976, see the third reference of (Bushell P J, 1990)).

$$
\operatorname{tr}(A B)^{k} \leq \operatorname{tr}\left(A^{k} B^{k}\right)
$$

In 1990, Bushell and Trustrum proved

$$
\sum_{i=1}^{n} \lambda_{i}^{k} \mu_{n-i+1}^{k} \leq \operatorname{tr}(A B)^{k} \leq \operatorname{tr}\left(A^{k} B^{k}\right) \leq \sum_{i=1}^{n} \lambda_{i}^{k} \mu_{i}^{k}
$$

Whereas the result proved by Lieb and Thirring, Bushell and Trustum only need to prove

$$
\sum_{i=1} \lambda_{i}^{k} \mu_{n-i+1}^{k} \leq \operatorname{tr}(A B)^{k} \leq \sum_{i=1} \lambda_{i}^{k} \mu_{i}^{k}
$$

They construct $B_{i}=U_{i} B U_{i}^{*}(i=1,2)$ in their proof firstly, then $\operatorname{tr}\left(A B_{1}\right)^{k}, \operatorname{tr}\left(A B_{2}\right)^{k}$ are the smallest and largest values of $\operatorname{tr}(A B)^{k}$, here $U_{i}$ is unitary matrix. The mistakes in their proof are mainly in the following two points:

(1) Exist unitary matrix $X$ with $\operatorname{rank} n$, such that $X^{*} A X, X^{*} B_{1} X, X^{*} B_{2} X$ become diagonal at the same time; (2)

$$
\operatorname{tr}(A B)^{k}=\sum_{i=1}^{n} \lambda_{\pi(i)}^{k} \mu_{i}^{k}
$$


We will point out that unitary matrix $X$ with rank $n$, which makes $X^{*} A X, X^{*} B_{1} X, X^{*} B_{2} X$ become diagonal at the same time does not definitely exist, and for general positive semi-definite Hermite matrix, (1) does also not definitely exist.

We give the following conclusions:

Exist unitary matrixX with rank $n$, such that $X^{*} A X$ and $X^{*} B_{1} X$ become diagonal at the same time; Exist unitary matrix $Y$ with rank $n$, such that $Y^{*} A Y$ and $Y^{*} B_{2} Y$ become diagonal at the same time. And for $B_{1}, B_{2}$,

$$
\begin{aligned}
& \operatorname{tr}\left(A B_{1}\right)^{k}=\sum_{i=1}^{n} \lambda_{\pi(i)}^{k} \mu_{i}^{k} \\
& \operatorname{tr}\left(A B_{2}\right)^{k}=\sum_{i=1}^{n} \lambda_{\pi(i)}^{k} \mu_{i}^{k}
\end{aligned}
$$

Thus complete the certification of Bushell-Trustrum inequality. We need to use the following Lemma:

Lemma (Wang Song-Gui, 2006, p. 143) Assuming that $\alpha_{1} \geq \cdots \geq \alpha_{n}, \mu_{1} \geq \cdots \geq \mu_{n}$. If $\pi(1), \cdots, \pi(n)$ is any permutation of $1, \cdots, n$, then

$$
\sum_{i=1}^{n} \alpha_{i} \mu_{n-i+1} \leq \sum_{i=1}^{n} \alpha_{\pi(i)} \mu_{i} \leq \sum_{i=1}^{n} \alpha_{i} \mu_{i}
$$

\section{Our proof}

Suppose $A>0, B>0$, otherwise for any $c>0$, There must be $A+c I>0, B+c I>0$, finally we take limit to the result obtained when $c \rightarrow 0$, then we conclude the proof.

Since entire unitary matrix with rank $n$ constitutes a closed set and mapping $U \rightarrow \operatorname{tr}\left(A U B U^{*}\right)^{k}$ is a continuous function defined on this closed set, so there must be the smallest and largest values in $U_{1}$ and $U_{2}$, Then

$$
\operatorname{tr}\left(A U_{2} B U_{2}^{*}\right)^{k} \leq \operatorname{tr}\left(A U B U^{*}\right)^{k} \leq \operatorname{tr}\left(A U_{1} B U_{1}^{*}\right)^{k}
$$

Especially, in (4), take $U=I$, then we have

$$
\operatorname{tr}\left(A U_{2} B U_{2}^{*}\right)^{k} \leq \operatorname{tr}(A B)^{k} \leq \operatorname{tr}\left(A U_{1} B U_{1}^{*}\right)^{k}
$$

If let $B_{i}=U_{i} B U_{i}^{*}(i=1,2)$, we will prove first: Exist unitary matrix $X$ with $\operatorname{rank} n$, such that $X^{*} A X$ and $X^{*} B_{1} X$ are diagonal.

Let

$$
\begin{aligned}
& R=\left(\begin{array}{cc}
R_{12} & 0 \\
0 & I
\end{array}\right) \\
& R=\left(\begin{array}{cc}
F_{12} & 0 \\
0 & 0
\end{array}\right)
\end{aligned}
$$

where

$$
\begin{gathered}
R_{12}=\left(1+|\varepsilon|^{2}\right)^{-\frac{1}{2}}\left[\begin{array}{cc}
1 & -\varepsilon \\
\bar{\varepsilon} & 1
\end{array}\right] \\
F_{12}=\frac{1}{|\varepsilon|}\left[\begin{array}{cc}
0 & -\varepsilon \\
\bar{\varepsilon} & 0
\end{array}\right]
\end{gathered}
$$

$\mathrm{R}, \mathrm{F}$ are $n \times n$ rectangular matrix, $0, \mathrm{I}$ are zero matrix and unit matrix on some degree.

Obviously, $\mathrm{R}$ is an unitary matrix, and to infinitely small $\varepsilon \neq 0$, $\mathrm{R}$ can denoted as

$$
R=I+|\varepsilon| F+o\left(|\varepsilon|^{2}\right)
$$

Here $o\left(|\varepsilon|^{2}\right)$ is $n \times n$ rectangular matrix, everyone of its element is infinitesimal of higher order of $|\varepsilon|$. For convenient we use $o\left(|\varepsilon|^{2}\right)$ to denote either matrix or number. 
In fact,

$$
\begin{gathered}
R-I-|\varepsilon| F=\left[\begin{array}{cc}
R_{12}-I-|\varepsilon| F_{12} & 0 \\
0 & 0
\end{array}\right] \\
R_{12}-I-|\varepsilon| F_{12}=\left[\begin{array}{cc}
\frac{1}{\sqrt{1+|\varepsilon|^{2}}}-1 & -\frac{\varepsilon}{\sqrt{1+|\varepsilon|^{2}}}-\varepsilon \\
\frac{\bar{\varepsilon}}{\sqrt{1+|\varepsilon|^{2}}}-\bar{\varepsilon} & \frac{1}{\sqrt{1+|\varepsilon|^{2}}}-1
\end{array}\right]
\end{gathered}
$$

From mathematical analysis, when $x \rightarrow 0$,

$$
1-\frac{1}{\sqrt{1+x^{2}}}=\frac{\sqrt{1+x^{2}}-1}{\sqrt{1+x^{2}}}=\frac{x^{2}}{\sqrt{1+x^{2}}\left(\sqrt{1+x^{2}}+1\right)} \sim x^{2}
$$

so elements in (11) and (12) are infinitesimal of higher order of $|\varepsilon|$, thus (10) holds. For any unitary matrix T, define

$$
\tilde{B}=\left(T R T^{*}\right) B\left(T R^{*} T^{*}\right)
$$

Since $\mathrm{R}$ is unitary matrix, $T R T^{*}$ is unitary matrix. Because $\mathrm{B}$ is positive semi-definite Hermite matrix, $T R^{*} T^{*}=$ $\left(T R T^{*}\right)^{*}, \tilde{B}$ is positive semi-definite Hermite matrix too. From (10), we get

$$
T R T^{*}=T\left(I+|\varepsilon| F+o\left(|\varepsilon|^{2}\right)\right) T^{*}=I+|\varepsilon| T F T^{*}+o\left(|\varepsilon|^{2}\right)
$$

Notice that $F^{*}=-F$,

$$
T R^{*} T^{*}=T\left(I+|\varepsilon| F^{*}+o\left(|\varepsilon|^{2}\right)\right) T^{*}=I-|\varepsilon| T F T^{*}+o\left(|\varepsilon|^{2}\right)
$$

Then

$$
\begin{aligned}
\tilde{B} & =B+|\varepsilon|\left(T F T^{*} B-B T F T^{*}\right)+o\left(|\varepsilon|^{2}\right) \\
& =B+|\varepsilon| T\left(F T^{*} B T-T^{*} B T F\right) T^{*}+o\left(|\varepsilon|^{2}\right) \\
& =B+|\varepsilon| T(F C-C F) T^{*}+o\left(|\varepsilon|^{2}\right)
\end{aligned}
$$

Here

$$
C=T^{*} B T
$$

It is easy to prove that for any two unitary matrix with rank $n \mathrm{P}$ and $\mathrm{Q}$, have

$$
\operatorname{tr}(P+|\varepsilon| Q)^{k}=\operatorname{tr} P^{k}+k|\varepsilon| \operatorname{tr} P^{k-1} Q+o\left(|\varepsilon|^{2}\right)
$$

Then from (16), (18)

$$
\begin{aligned}
\operatorname{tr}(A \tilde{B})^{k} & =\operatorname{tr}\left(A B+|\varepsilon| A T(F C-C F) T^{*}+o\left(|\varepsilon|^{2}\right)\right)^{k} \\
& =\operatorname{tr}(A B)^{k}+k|\varepsilon| \operatorname{tr}(A B)^{k-1} A T(F C-C F) T^{*}+o\left(|\varepsilon|^{2}\right) \\
& =\operatorname{tr}(A B)^{k}+k|\varepsilon| \operatorname{tr}[D(F C-C F)]+o\left(|\varepsilon|^{2}\right)
\end{aligned}
$$

Here

$$
D=T^{*}(A B)^{k-1} A T
$$

We can prove that $(A B)^{k-1} A \geq 0$

In fact, notice that $\mathrm{A}$ and $\mathrm{B}$ are both positive semi-definite Hermite matrixes.

When $k=2, A B A=A B^{\frac{1}{2}} B^{\frac{1}{2}} A=\left(B^{\frac{1}{2}} A\right)^{*} B^{\frac{1}{2}} A \geq 0$.

When $k=3, A B A B A=A B A^{\frac{1}{2}} A^{\frac{1}{2}} B A=\left(A^{\frac{1}{2}} B A\right)^{*} A^{\frac{1}{2}} B A \geq 0$. It can be proved by induction.

$\operatorname{In}(20)$, because $(A B)^{k-1} A$ is positive semi-definite, $\mathrm{T}$ is any unitary matrix, so we can choose unitary matrix $\mathrm{T}$, such that $\mathrm{D}$ becomes diagonal,

$$
D=\operatorname{diag}\left(d_{1}, \cdots, d_{n}\right), \quad d_{1} \geq \cdots \geq d_{n} \geq 0
$$


Let $C=\left(\begin{array}{cc}C_{1} & C_{2} \\ C_{3} & C_{4}\end{array}\right), D=\left(\begin{array}{cc}D_{1} & 0 \\ 0 & D_{2}\end{array}\right)$, where $C_{1}=\left(\begin{array}{cc}C_{11} & C_{12} \\ C_{21} & C_{22}\end{array}\right), D_{1}=\left(\begin{array}{cc}d_{1} & 0 \\ 0 & d_{2}\end{array}\right)$; Notice that $C_{1}$ is Hermite matrix, $F_{12}^{*}=-F_{12}$, then

$$
\begin{aligned}
|\varepsilon| \operatorname{tr} D(F C-C F) & =|\varepsilon| \operatorname{tr}\left[\begin{array}{cc}
D_{1} & 0 \\
0 & D_{2}
\end{array}\right]\left[\left(\begin{array}{cc}
F_{12} & 0 \\
0 & 0
\end{array}\right)\left(\begin{array}{cc}
C_{1} & C_{2} \\
C_{3} & C_{4}
\end{array}\right)-\left(\begin{array}{cc}
C_{1} & C_{2} \\
C_{3} & C_{4}
\end{array}\right)\left(\begin{array}{cc}
F_{12} & 0 \\
0 & 0
\end{array}\right)\right] \\
& =|\varepsilon| \operatorname{tr}\left[\begin{array}{cc}
D_{1} & 0 \\
0 & D_{2}
\end{array}\right]\left[\begin{array}{cc}
F_{12} C_{1}-C_{1} F_{12} & F_{12} C_{2} \\
-C_{2} F_{12} & 0
\end{array}\right] \\
& =|\varepsilon| \operatorname{tr}\left[\begin{array}{cc}
D_{1}\left(F_{12} C_{1}-C_{1} F_{12}\right) & D_{1} F_{12} C_{2} \\
-D_{2} C_{2} F_{12} & 0
\end{array}\right] \\
& =|\varepsilon| \operatorname{tr} D_{1}\left(F_{12} C_{1}-C_{1} F_{12}\right) \\
& =|\varepsilon| \operatorname{tr} D_{1}\left(F_{12} C_{1}+\left(F_{12} C_{1}\right)^{*}\right) \\
& =\left(d_{2}-d_{1}\right)\left(\bar{\varepsilon} c_{12}+\varepsilon \bar{c}_{12}\right)
\end{aligned}
$$

The last equation is right because $C_{1}$ is Hermite matrix, and

$$
|\varepsilon| F_{12} C_{1}=\left(\begin{array}{cc}
0 & -\varepsilon \\
\bar{\varepsilon} & 0
\end{array}\right)\left(\begin{array}{cc}
c_{11} & c_{12} \\
c_{21} & c_{22}
\end{array}\right)=\left(\begin{array}{cc}
-\varepsilon c_{21} & -\varepsilon c_{22} \\
\bar{\varepsilon} c_{11} & \bar{\varepsilon} c_{12}
\end{array}\right)
$$

By (22) and (19), then

$$
\operatorname{tr}(A \tilde{B})^{k}-\operatorname{tr}(A B)^{k}=k\left(d_{2}-d_{1}\right)\left(\bar{\varepsilon} c_{12}+\varepsilon \bar{c}_{12}\right)+o\left(|\varepsilon|^{2}\right)
$$

This formula is correct on arbitrary semi-positive Hermite matrix B and infinitely small $\varepsilon \neq 0$.

Especially, set $B=B_{1}, \varepsilon=\eta c_{12}, \eta>0$, If $d_{2} \neq d_{1}$, then by definition of $B_{1}$ and (2), we obtain $\bar{\varepsilon} c_{12}+\varepsilon \bar{c}_{12}=$ $\eta\left|c_{12}\right|^{2}=0$, then $c_{12}=\bar{c}_{21}=0$.

Similarly, we take R, F such that their $i, j(i<j)$ row and column have form of (8), (9), and similar to the proof above, then it can be obtained.

$$
\operatorname{tr}(A \tilde{B})^{k}-\operatorname{tr}(A B)^{k}=k\left(d_{j}-d_{i}\right)\left(\bar{\varepsilon} c_{i j}+\varepsilon \bar{c}_{i j}\right)+o\left(|\varepsilon|^{2}\right)
$$

Set $\varepsilon=\eta c_{i j}, \eta>0$, Use the same method $c_{i j}=\bar{c}_{j i}=0$ can be obtained.

Suppose $c_{1}>c_{2}>\cdots>c_{l}$ are $l$ different value of $d_{1}, \cdots, d_{n}$, here $D=\operatorname{diag}\left(c_{1} I_{n 1}, \cdots c_{l} I_{n l}\right)$. make $C=T^{*} B_{1} T$ become block matrix

$$
C=\operatorname{diag}\left(C_{1}, \cdots, C_{l}\right)
$$

Here $C_{i}$ is positive semi-definite Hermite matrix with rank $n_{i}$. Let $V_{i}(i=1, \cdots, l)$ is unitary matrix, such that $E_{i}=V_{i}^{*} C_{i} V_{i}, i=1, \cdots, l$, becomes diagonal matrix.

Let

$$
\begin{aligned}
& V=\operatorname{diag}\left(V_{1}, \cdots, V_{l}\right) \\
& E=\operatorname{diag}\left(E_{1}, \cdots, E_{l}\right)
\end{aligned}
$$

Set $X=T V$, then $X$ is an unitary matrix, and

$$
X^{*} B_{1} X=V^{*} T^{*} B_{1} T V=V^{*} C V=E
$$

This is a diagonal matrix, its diagonal elements are eigenvalues of $B_{1}$, furthermore

$$
X^{*}\left(A B_{1}\right)^{k-1} A X=V^{*} T^{*}\left(A B_{1}\right)^{k-1} A T V=V^{*} D V=D
$$

The last equation is correct because V and D are block matrix with same degree. By (28) and (29) we know

$$
\left(E^{\frac{1}{2}}\left(X^{*} A X\right) E^{\frac{1}{2}}\right)^{k}=E^{\frac{1}{2}} X^{*}\left(A B_{1}\right)^{k-1} A X E^{\frac{1}{2}}=E^{\frac{1}{2}} D E^{\frac{1}{2}}
$$


then

$$
X^{*} A X=E^{-\frac{1}{2}}\left(E^{\frac{1}{2}} D E^{\frac{1}{2}}\right)^{\frac{1}{k}} E^{-\frac{1}{2}}
$$

It is a diagonal matrix. It be proved that exist $n \times n$ unitary matrix such that $X^{*} A X, X^{*} B_{1} X$ are all diagonal matrix.

Similarly, in(24), let $B=B_{2}, \varepsilon=\eta c_{i j}, \eta<0$, then $c_{i j}=\bar{c}_{j i}=0$. Notice that because $C=T^{*} B T$ and $B_{i}$ are different, so we write as $G=T^{*} B_{2} T$.

Suppose that $g_{1}>g_{2}>\cdots>g_{m}$ are $m$ different values of $d_{1}, \cdots, d_{n}$, here $D=\operatorname{diag}\left(g_{1} I_{n 1}, \cdots g_{m} I_{n_{m}}\right)$, make $G=T^{*} B_{2} T$ become block matrix

$$
G=\operatorname{diag}\left(G_{1}, G_{2}, \cdots G_{m}\right)
$$

Let $W_{i}(i=1,2, \cdots m)$ be an unitary matrix, such that $W_{i}^{*} G_{i} W_{i}(i=1,2, \cdots m)$ is diagonal matrix. Write

$$
W=\operatorname{diag}\left(W_{1}, W_{2}, \cdots W_{m}\right)
$$

Set $Y=T W$, then $\mathrm{Y}$ is an unitary matrix, similar to the proof on (28)-(31), it be obtained that exist unitary matrix $Y$ such that $Y^{*} A Y, Y^{*} B_{2} Y$ are all diagonal matrix.

According to (28) and (31), $X^{*} A X, X^{*} B_{1} X, Y^{*} A Y, Y^{*} B_{2} Y$ are all diagonal matrixes. Notice that $X^{*} B_{1} X=$ $X^{*} U_{1} B_{1} U_{1}{ }^{*} X, Y^{*} B_{2} Y=Y^{*} U_{2} B_{2} U_{2}{ }^{*} Y, U_{1}, U_{2}, X, Y$ are all unitary matrix, so diagonal elements of $X^{*} B_{1} X, Y^{*} B_{2} Y$ are eigenvalues of $B$. Thus

$$
\begin{aligned}
& \operatorname{tr}\left(A B_{1}\right)^{k}=\operatorname{tr}\left(X^{*}\left(A B_{1}\right)^{k} X\right)=\operatorname{tr}\left(X^{*} A X X^{*} B_{1} X\right)^{k}=\sum_{i=1}^{n} \lambda_{\pi(i)}^{k} \mu_{i}^{k} \\
& \operatorname{tr}\left(A B_{2}\right)^{k}=\operatorname{tr}\left(Y^{*}\left(A B_{2}\right)^{k} Y\right)=\operatorname{tr}\left(Y^{*} A Y Y^{*} B_{2} Y\right)^{k}=\sum_{i=1}^{n} \lambda_{\pi^{\prime}(i)}^{k} \mu_{i}^{k}
\end{aligned}
$$

Here $\pi(i), \pi^{\prime}(i)$ is any permutation of $1,2, \cdots, n$, respectively. From Lemma,

$$
\begin{gathered}
\operatorname{tr}\left(A B_{1}\right)^{k} \leq \sum_{i=1}^{n} \lambda_{i}^{k} \mu_{i}^{k} \\
\operatorname{tr}\left(A B_{2}\right)^{k} \geq \sum_{i=1}^{n} \lambda_{i}^{k} \mu_{n-i+1}^{k}
\end{gathered}
$$

And using(5), then the proof is completed.

\section{References}

Bushell P J, Trustrum G B. (1990). Trace inequalities for positive definite matrix power products. Linear Algebra and its Applications, 132, 173-178.

Marcus M, An eigenvalue inequality for the product of normal matrices. Amer Math Monthly, (1956), 63:173174.

Marshall A W and Olkin I. Inequalities: Majorization and its applications. Academic, New York, (1979).

Wang Song-Gui, Wu Mi-Xia and Jia Zhong-Zhen. (2006). Matrix Inequalities, Beijing: Science Press. 